\title{
Plasma steroid metabolome profiling for the diagnosis of adrenocortical carcinoma
}

\section{Sophie Schweitzer1,*, Meik Kunz 2,3,4,*, Max Kurlbaum ${ }^{1,5}$, Johannes Vey²,3, Sabine KendI', Timo Deutschbein', Stefanie Hahner ${ }^{1,3}$, Martin Fassnacht ${ }^{1,3,5}$, Thomas Dandekar ${ }^{2,3}$ and Matthias Kroiss , $^{1,3,5}$}

${ }^{1}$ Division of Endocrinology/Diabetology and Core Unit Clinical Mass Spectrometry, Department of Internal Medicine I, University Hospital Würzburg, ${ }^{2}$ Department of Bioinformatics, Biocenter, University of Würzburg, ${ }^{3}$ University of Würzburg, Comprehensive Cancer Center Mainfranken, ${ }^{4}$ Chair of Medical Informatics, Friedrich-Alexander University of Erlangen-Nürnberg, Erlangen, Germany, and ${ }^{5}$ University Hospital Würzburg, Central Laboratory, Core Unit Clinical Mass Spectrometry, Würzburg, Germany

*(S Schweitzer and M Kunz contributed equally to this work)

Correspondence should be addressed to M Kroiss or M Kunz Email

Kroiss_M@ukw.de or meik. kunz@uni-wuerzburg.de

\section{Abstract}

Objective: Current workup for the pre-operative distinction between frequent adrenocortical adenomas (ACAs) and rare but aggressive adrenocortical carcinomas (ACCS) combines imaging and biochemical testing. We here investigated the potential of plasma steroid hormone profiling by liquid chromatography tandem mass spectrometry (LC-MS/MS) for the diagnosis of malignancy in adrenocortical tumors.

Design: Retrospective cohort study of prospectively collected EDTA-plasma samples in a single tertiary reference center.

Methods: Steroid hormone profiling by liquid chromatography tandem mass spectrometry (LC-MS/MS) in random plasma samples and logistic regression modeling.

Results: Fifteen steroid hormones were quantified in 66 ACAs (29 males; M) and 42 ACC (15 M) plasma samples.

Significantly higher abundances in ACC vs ACA were observed for 11-deoxycorticosterone, progesterone,

17-hydroxyprogesterone, 11-deoxycortisol, DHEA, DHEAS and estradiol (all $P<0.05$ ). Maximal areas under the curve (AUC) for discrimination between ACA and ACC for single analytes were only 0.76 (estradiol) and 0.77 (progesterone), respectively. Logistic regression modeling enabled the discovery of diagnostic signatures composed of six specific steroids for male and female patients with AUC of 0.95 and 0.94, respectively. Positive predictive values in males and females were 92 and 96\%, negative predictive values 90 and $86 \%$, respectively.

Conclusion: This study in a large adrenal tumor patient cohort demonstrates the value of plasma steroid hormone profiling for diagnosis of ACC. Application of LC-MS/MS analysis and of our model may facilitate diagnosis of malignancy in non-expert centers. We propose to continuously evaluate and improve diagnostic accuracy of LC-MS/MS profiling by applying machine-learning algorithms to prospectively obtained steroid hormone profiles.

\section{Introduction}

Adrenal tumors are frequent neoplasms in humans and increasingly detected upon imaging that was not performed for suspected adrenal disease. Radiological studies report a prevalence of such 'adrenal incidentalomas' of 3-10\% (1). The majority of these tumors are non-functioning adrenocortical adenomas (ACAs) (1). Current workup includes radiological and endocrinological workup with the aim to reliably distinguish between ACA and rare but often aggressive adrenocortical carcinoma (ACC) or lesions of non-adrenocortical origin and detect hormone excess $(2,3)$. 
Evidence of high fat content demonstrated by Hounsfield units below 10 in unenhanced computed tomography is consistent with a benign tumor (4). Although less robust, comparable criteria are relative and absolute washout of $>40 \%$ and $60 \%$ in contrastenhanced computed tomography (5) and loss of magnetic resonance imaging signal in opposed phase (6). Diagnostic uncertainty exists when fat content is low, which is the case in at least $30 \%$ of cases. Although recent guidelines on adrenal incidentalomas are aiming to reduce radiological follow-up (1), many patients still receive repeated imaging. Besides, unsettledness of affected patients and care providers results in frequently unjustified adrenalectomy. Whereas surgery may unnecessarily expose patients with ACA to the risk of surgery and increase costs for health care systems, repeated imaging likely increases the psychological burden for patients.

Although ACC is an exceedingly rare tumor with an annual incidence of only $\sim 0.5-2 /$ million inhabitants ( 7 , 8), aggressive tumor biology leads to a dismal prognosis in many cases. Rapid diagnosis is therefore essential not only to ensure timely therapeutic intervention but also to avoid tumor progression (1). Complete surgical removal is the treatment of choice in localized disease, and mitotane is often advocated as an adjuvant treatment (9, 10). In unresectable or advanced stages, mitotane alone (11) or in combination with etoposide, doxorubicin and cisplatin (3) is currently considered as the treatment of choice. However, objective treatment response is around $20-25 \%$ only and disease stabilization often short-lived which leads to median overall survival between 12 and 15 months in metastatic disease.

Some decades ago, it has already been considered to exploit variabilities of steroidogenic hormone production as markers of malignancy in adrenal masses $(12,13)$. More recently, it has been shown that steroid profiling by gas chromatography mass spectrometry (GC-MS) of urine samples collected over $24 \mathrm{~h}$ has diagnostic value in discriminating benign and malignant adrenocortical tumors $(14,15)$.

However, GC-MS is relatively time consuming and still unavailable in most centers. In addition, urine collection is prone to sampling errors that result in false steroid quantification.

In contrast, liquid chromatography tandem mass spectrometry (LC-MS/MS) is a much faster method and available in an increasing number of centers. This analytical technique overcomes many limitations that are usually associated with immunoassays and GC-MS $(16,17)$ while enabling rapid quantitation of small molecules with high specificity and sensitivity. Recently, commercially available internal standards, calibrators and validated controls have become available. Some manufacturers offer kit solutions that comply with applicable regulations for in vitro diagnostics (e.g. European In-Vitro-Diagnostic Regulation, IVDR) which likely will facilitate access to this technology further, especially for routine patient care.

In a very small study with only ten ACC patients, Taylor et al. suggested that serum steroid panel analysis may help in the differential diagnosis of ACC and nonACC adrenal tumors (e.g. ACA) (18) and similar panel analyses have been successfully conducted in cortisolproducing adenomas (19). LC-MS/MS analysis of serum or plasma samples would not only facilitate sampling but also speed up laboratory diagnostics and thereby facilitate the difficult differential diagnosis. So far however both the common dilemma of limited sample size and equivocal results of multiple single steroids prevent the direct application of this method in daily diagnostic routine.

This led us to evaluate the diagnostic value of a 15-steroid plasma panel in a large series of ACA and ACC patients, including bioinformatic modeling.

\section{Patients and methods}

\section{Study population}

Plasma samples were prospectively collected in a single center as part of the European Network for the study of adrenal tumors (ENSAT) database and biorepository which has been approved by the Local Ethics Committee of the University of Würzburg (approval numbers 86/03 and $88 / 11$, respectively). All patients provided written informed consent.

EDTA plasma samples were obtained during routine blood sampling in the morning, centrifuged and stored at $-80^{\circ} \mathrm{C}$ as part of the Interdisciplinary Bank of Biomaterials and Data of the University Hospital of Würzburg and the Julius Maximilian University of Würzburg (IBDW). Consecutive samples from patients with non-aldosteroneproducing ACA and ACC were chosen for analysis. Definition of hormone excess was judged after clinical workup at our center and on the basis of routine laboratory testing with serum androstenedione, DHEAS and serum estradiol (in males), aldosterone to renin ratio and 24-h urinary free cortisol as well as $1 \mathrm{mg}$ dexamethasone suppression test (DST). For definition of glucocorticoid excess, we applied the criteria proposed by the ESE/ENSAT incidentaloma guideline (1). Patients with autonomous 
cortisol secretion ( $1 \mathrm{mg}$ DST cortisol $>5 \mu \mathrm{g} / \mathrm{dL}$ ) and possible autonomous cortisol secretion $(>1.8-4.9 \mu \mathrm{g} / \mathrm{dL})$ were considered as cortisol-producing tumors. Tumors with no evidence of hormone secretion were considered inactive. The underlying diagnosis was ascertained by histology; in non-operated ACA patients, the recent guidelines were applied (1) to exclude malignancy. ACC patients currently treated with mitotane, ketoconazole, metyrapone or mifepristone were excluded.

\section{Steroid hormone analysis}

Liquid chromatography mass spectrometry tandem mass spectrometry was performed with a Sciex 6500+ QTRAP (SCIEX, Framingham, USA) MS-system linked with an Agilent 1290 HPLC-system (G4226A autosampler, infinityBinPump, G1316C column-oven, G1330B thermostat). Analysis was performed with the commercially available MassChrom-Steroids in Serum/Plasma kit (Chromsystems, Gräfelfing) which permits the quantification of 15 steroid parameters (MRM-Mode) and corresponding isotope standards according to the manufacturer's instruction. After offline solid phase extraction of $500 \mu \mathrm{L}$ serum, $15 \mu \mathrm{L}$ were used for analysis. Concentrations were calculated with Analyst Software (1.6.3) via 6 point calibration and $1 / \times$ weighting. Correctness of measurements was controlled by commercial quality controls and periodic participation in ring trails. Analytes measured were aldosterone, androstenedione, 11-deoxycortisol, 11-deoxycorticosterone, 21-deoxycortisol, cortisol, cortisone, corticosterone, DHEA, DHEAS, DHT, estradiol, progesterone, 17-hydroxyprogesterone (17-OHP) and testosterone.

Methodological details are provided in Supplementary Methods available online at https://doi.org/10.17605/ OSF.IO/8YGNK, see section on supplementary data given at the end of this article).

\section{Statistical analysis}

Statistical analyses were performed using SPSS version 24 (IBM Corp.) and R version 3.4.4 (R Core Team and contributors worldwide). Data were analyzed using the Wilcoxon rank-sum test (also called Mann-Whitney $U$ test), chi-square test for categorical variables and a logistic regression analysis. Values below lower limit of quantification (LLOQ) were set to $0.5 \times$ LLOQ.

Logistic regression analyses were performed using the glm function from the stats package ( $\mathrm{R}$ Core Team and contributors worldwide). In addition, a model selection based on the Akaike Information Criteria (AIC) was applied, using the stepAIC function from the MASS package (20). This value is a quality indicator of how good the statistical model describes the data.

The following stepwise regression methods were used $(21,22)$ : forward selection (starts with a null model and iteratively adds the steroid with the best AIC), backward selection (or backward elimination; starts with a full model of 15 steroids and the steroid with highest AIC is iteratively removed) and stepwise selection (combination of forward and backward selection, in which the lowest/ highest AIC is sequentially added and removed). As initial step, the null and full logistic regression models were built, and subsequently the stepwise model selection was performed.

Receiver operating characteristics (ROC) curves and area under the curve (AUC) values were generated using the functions prediction and performance from the ROCR package (23). In addition, a confusion matrix was calculated for each signature (as a measure of diagnostic performance to classify each patient into the ACC and ACA groups), using the confusion matrix function from the SDMTools package (24). Subsequently, the following diagnostic parameters were calculated:

- Sensitivity: $t_{p} / t_{p}+f_{n}$

- Specificity: $t_{n} / t_{n}+f_{p}$

- Positive predictive value (PPV, precision): $t_{p} / t_{p}+f_{p}$

- Negative predictive value (NPV): $t_{n} / t_{n}+f_{n}$

- Accuracy (correct classification rate): $t_{p}+t_{n} / t_{p}+f_{p}+t_{n}+f_{n}$ $\left(t_{p}=\right.$ true positive, $f_{p}=$ false positive, $f_{n}=$ false negative, $t_{n}=$ true negative).

\section{Results}

\section{Clinical characteristics}

Demographic and clinical characteristics of the patients are provided in Table 1. Age was comparable between groups. Female sex was overrepresented in both the ACA and ACC groups. Sixty percent of ACC patients had localized (ENSAT stage I-III) disease.

\section{Steroid hormone profiling}

Overall, ACC patients had significantly higher concentrations (nmol/L, median) of precursor hormones (progesterone: 0.080 vs $0.555, \quad P<0.001$; 17-hydroxyprogesterone: 1.132 vs $2.100, \quad P=0.005$; 
Table 1 Demographic and clinical characteristics of the adrenal tumor patients. Data are presented as $n(\%)$ or $n$ (range). Comparison of categorical variables between groups was conducted using the Chi-Square test.

\begin{tabular}{|c|c|c|c|}
\hline & ACA $(n=66)$ & $\overline{A C C}(n=42)$ & $\boldsymbol{P}$ \\
\hline Sex, male & $29(44)$ & $15(36)$ & 0.396 \\
\hline $\begin{array}{l}\text { Age at time of sample }{ }^{\dagger} \\
\text { collection; years, median } \\
\text { (range) }\end{array}$ & $58(21-81)$ & $57(20-80)$ & \\
\hline \multicolumn{4}{|l|}{ Hormone secretion } \\
\hline Inactive & $42(64)$ & $16(38)$ & \\
\hline Endocrine active & $24(36)$ & $26(62)$ & $0.009^{a}$ \\
\hline Cortisol only & $24(36)$ & $7(17)$ & $0.607^{b}$ \\
\hline Androgens & & $4(10)$ & \\
\hline $\begin{array}{l}\text { Cortisol and } \\
\text { mineralocorticoids }\end{array}$ & & $2(5)$ & \\
\hline Cortisol and sex steroids & & $12(29)$ & \\
\hline Estrogens & & $1(2)$ & \\
\hline \multicolumn{4}{|l|}{ ENSAT stage at initial diagnosis } \\
\hline I & & $4(10)$ & \\
\hline ॥ & & $14(33)$ & \\
\hline III & & $7(17)$ & \\
\hline IV & & $17(40)$ & \\
\hline \multicolumn{4}{|l|}{ Tumor present at sampling } \\
\hline Primary tumor & $66(100)$ & $13(31)$ & \\
\hline Primary tumor + metastases & & $11(26)$ & \\
\hline Local recurrence & & $3(7)$ & \\
\hline Distant recurrence & & $9(21)$ & \\
\hline Local + distant recurrence & & $6(14)$ & \\
\hline
\end{tabular}

11-deoxycortisol: 1.994 vs $3.035, P=0.01)$, sex steroids (DHEA: 2.674 vs 5.434, $P=0.002$; DHEAS: 1469 vs 3279, $P=0.001$; estradio:, 0.055 vs $0.219, P<0.001)$, mineralocorticoids (11-deoxycorticosterone: 0.081 vs $0.295, P<0.001)$ and cortisol (269.1 vs $378.1, P=0.015)$.

Separate comparison of clinically inactive ACA $(n=42)$ and ACC $(n=16)$ revealed significant differences for estradiol( 0.055 vs $0.055, P=0.018)$, 11-deoxycorticosterone (0.048 vs $0.101, P=0.046)$ and progesterone (0.080 vs 0.169, $P=0.015)$. Similarly, ACA and ACC with clinical or biochemical glucocorticoid excess showed higher concentrations of cortisol (379.5 vs 539.4, $P=0.004)$, 11-deoxycortisol (2.101 vs 8.121, $P<0.001$ ), progesterone (0.080 vs $0.703, P<0.001), 17 \mathrm{OH}$-progesterone $(0.883$ vs $4.636, P<0.001)$, 11-deoxycorticosterone $(0.110$ vs $0.521, P=0.007)$, DHEA (1.610 vs 5.899-, $P=0.01)$, DHEAS (775.1 vs $5257.4, P=0.002)$ and estradiol (0.055 vs 0.228 , $P<0.001)$ in ACC vs ACA patients.

Subsequently, we analyzed the steroid profile separately for both sexes (Fig. 1 and Table 2). While 11-deoxycorticosterone, progesterone and estradiol
A Plasma steroid concentration in male patients $(\mathrm{nmol} / \mathrm{l})$

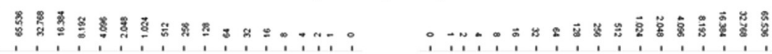

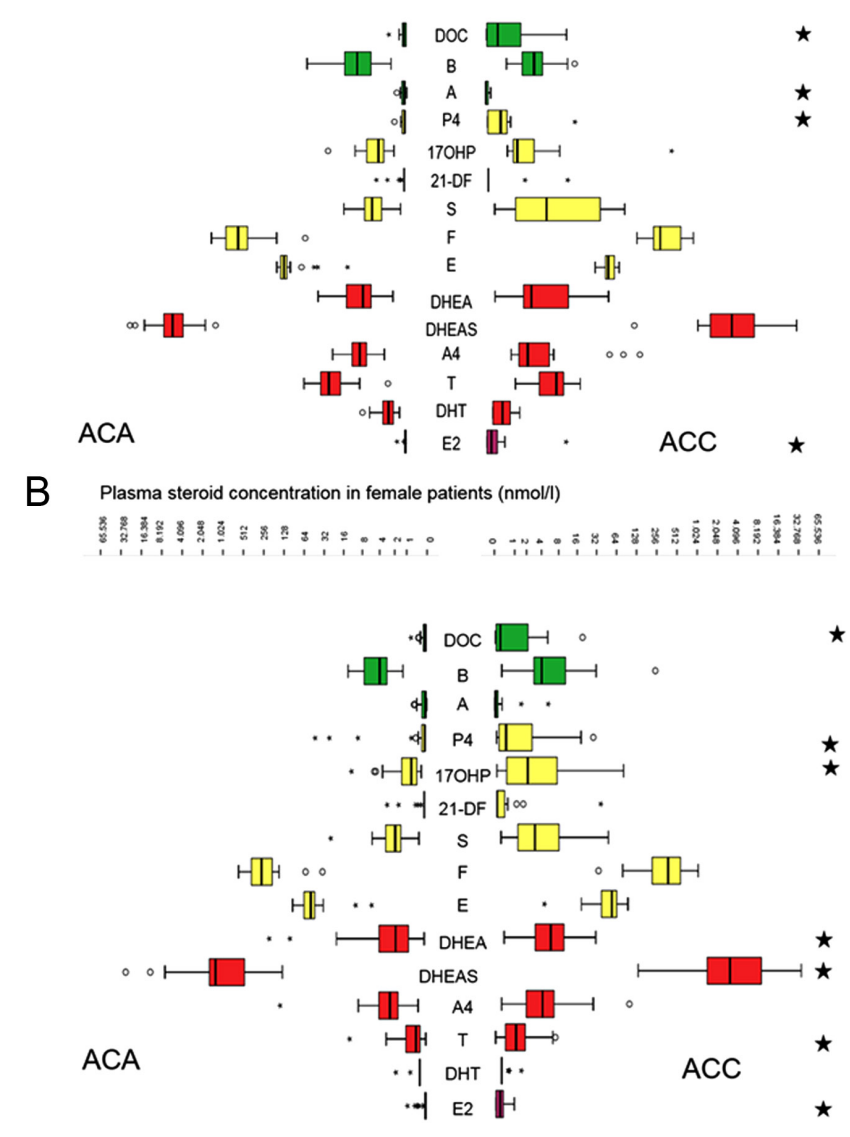

Figure 1

Sex-specific steroid hormone patterns in ACA and ACC patients. Log2-transformed absolute quantity of the 15 steroid hormones 11-deoxycorticosterone (DOC), corticosterone (B), aldosterone (A4), progesterone (P4), 17OHP, 21-deoxycortisol (21-DF), 11-deoxycortisol (B), cortisol (F), cortisone (E), DHEA, DHEAS, androstenedione (A4), testosterone, DHT and estradiol (E2) as measured by LC-MS/MS in non-aldosterone-producing adrenocortical adenomas (ACAs) and adrenocortical carcinoma (ACC) patients. (A) In male, significantly increased quantities of 11DOC, A, P4, B and E2 were observed in ACC (right panel, $n=15)$ vs ACA left panel $(n=29)$. (B) Female patients showed increased 11DOC, P4, 17-OHP, DHEA, DHEAS and testosterone between ACC $(n=27)$ and ACA $(n=37)$. Steroids are color coded in green for mineralocorticoids and precursors, yellow glucocorticoids and precursors, red androgens and gray estradiol. The asterisks indicate $P<0.05$.

were significantly different in both males and females (all $P<0.05)$, aldosterone was lower in males $(P=0.002)$; 17OH-progesterone $(P=0.015)$, DHEA $(P=0.002)$, DHEAS 
$(P=0.001)$ and testosterone $(P=0.027)$ were elevated in females (ACC vs ACA patients).

Overall, most analytes exhibited marked overlap between ACA and ACC samples, thereby leading to poor diagnostic performance of individual steroids. ROC analysis of individual steroid hormones exhibited overall unsatisfactory diagnostic power, with highest AUC values of 0.76 (M: 0.805; F: 0.722) and 0.77 (M: 0.767; F: 0.754) for estradiol and progesterone, respectively (Supplementary Fig. 1, available online at https://doi.org/10.17605/OSF. IO/8YGNK).

\section{Logistic regression modeling}

Steroid combinations were selected based on the lowest AIC and highest AUC values. Given the sex-specific differences in steroid hormone abundance between ACA and ACC in our previous comparison, we decided to separately optimize the models for male and female patients.

Males

Stepwise forward selection (model selection starting with null model) identified a three steroid signature containing corticosterone, estradiol and progesterone with lowest AIC (34.14) and highest AUC (0.88) values (Supplementary Fig. 2A and C, available online at https://doi.org/10.17605/ OSF.IO/8YGNK). The forward model selection resulted in a four-steroid signature (corticosterone, estradiol, progesterone and aldosterone) with AIC value of 35.58 and AUC of 0.90 (Supplementary Fig. 2B and C). On the other hand, the stepwise backward selection (model selection starting with full model) and backward model selection resulted in a six steroid signature (corticosterone, estradiol, progesterone, 11-deoxycortisol, DHEA, androstenedione) with AIC value of 34.48, but the highest AUC (0.95) of all models was tested (Fig. 2A and B). For these models, a confusion matrix and diagnostic parameters (sensitivity, specificity, NPV, PPV and accuracy) were calculated. The six steroid signature (corticosterone, estradiol, progesterone, 11-deoxycortisol, DHEA, androstenedione) showed the best predictive parameters in males (Fig. 2C).

\section{Females}

The stepwise forward and stepwise backward selection resulted in a six steroid signature (DHEAS, androstenedione, DHEA, DHT, 11-deoxycorticosterone, aldosterone) with the lowest AIC (49.91) and the highest AUC (0.95) value 

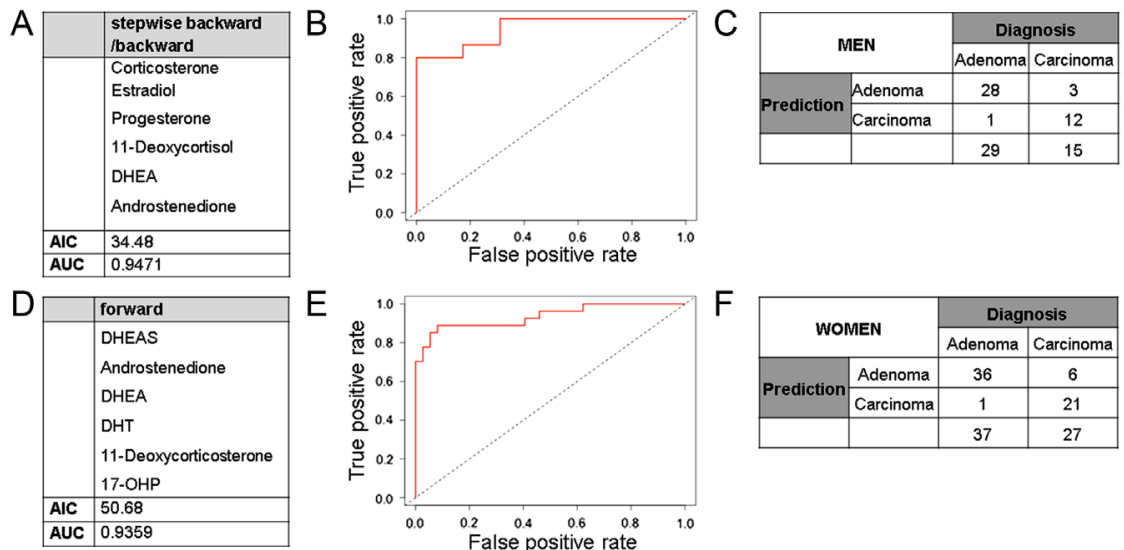

\section{Figure 2}

Optimal diagnostic signatures developed by logistic regression modeling. Separate models for the diagnosis of ACC were developed for male (A, B and C) and female (D, E and F) patients with adrenal tumors. (A) and (D) provide the list of hormones with diagnostic value that have been identified by the model optimization indicated. Akaike Information Criterion (AIC) and area under the curve (AUC) are given. (B) and (E) show receiver operator characteristics (ROC) curved of steroid signatures. (C) and (F) provide the confusion matrix of the selected models: PPV, positive predictive value; NPV, negative predictive value, each for diagnosis of ACC.

(Supplementary Fig. 3A and B). Backward selection led to a five steroid signature (DHEAS, androstenedione, DHEA, DHT, 11-deoxycorticosterone) with AIC value of 49.92 and AUC of 0.93 (Supplementary Fig. 3C and D).

The forward selection led us to a slightly different six steroid signature which comprised 17-OHP but not aldosterone (DHEAS, androstenedione, DHEA, DHT, 11-deoxycorticosterone, 17-OHP) and described the data slightly less well with an AIC of 50.68 and AUC of 0.94 . However, this signature resulted in the highest PPV and NPV values in females (Fig. 2F).

To determine whether the development of a nonsex-specific model could achieve similar performance, we applied the same modeling strategy to the entire series of samples regardless of sex. However, independent of the model selection, AIC values were at best 91.38 for the forward and stepwise backward selection models, which resulted both in a six steroid signature of DHEAS, DHEA, testosterone, DHT, 11-deoxycorticosterone and aldosterone. All sex-independent models had lower PPV and NPV.

The script of the logistic regression modeling is provided online at at https://doi.org/10.17605/OSF. IO/8YGNK.

\section{Discussion}

Analysis of steroid hormones by gas chromatography mass spectrometry (GC-MS) in urine samples is a useful tool for the diagnosis of steroid synthesis disorders such as congenital adrenal hyperplasia $(25,26,27,28)$ and P450 oxidoreductase deficiency $(27,29)$. It has also been successfully applied for the discrimination between benign and malignant adrenal masses (14, 30, 31, 32).

We here demonstrate that plasma steroid hormone profiling may have similar diagnostic potential.

Theoretically, the usage of 24-h urine as a matrix has the advantage over random plasma samples to also include the information about diurnal rhythmicity. However, 24-h urine collections are cumbersome because they require clear instruction and thorough cooperation of patients. Sampling errors are therefore a relevant clinical problem especially in the outpatient setting that some try to account for by measuring creatinine as a plausibility check or for normalization.

As a diagnostic technology, LC-MS/MS provides the advantage of much more rapid results and simpler sample preparation (e.g. no need for derivatization steps) compared to GC-MS. The LC-MS/MS method used here permits the acquisition of 15 steroids within 22 min while maintaining the high specificity of mass spectrometry and exact quantitation by the utilization of isotope standards. The utilization of a commercially available method that complies with applicable in vitro diagnostics regulation from our point of view renders the application of LC-MS/ MS assays in routine patient care straightforward and may promote the usage of steroid hormone profiles for the differential diagnosis of adrenal tumors. The advantage 
of a single blood sample over an error-prone 24-h urine collection is obvious. Nevertheless, many established GC-MS applications allow the detection of a broader spectrum of steroid hormones and maintain their value for specific clinical questions.

In comparison with the GC-MS study by Arlt et al. (14), the AUC values of our models that comprise six steroid hormones are comparably smaller than those obtained with nine steroid metabolites in 24-h urine, which was 0.96. Inclusion of all measured plasma steroids did not improve the model (Supplementary Fig. 4). It may be that this is in part due to the larger proportion of advanced stage tumors in the series by Arlt et al., which may be associated with more prominent alterations of steroid hormones and precursors. Although that study also includes a larger number of ACA, we think that a final conclusion regarding the optimal matrix and method of analysis cannot be drawn yet.

Similar to investigations by GC-MS in 24-h urine (14, $30,31,32$ ), we find that steroid hormone precursors and androgens are increased in ACC compared to ACA, and this was true for both endocrine active and hormonally silent tumors. This finding is in line with the notion that ACC dispose of an inefficient steroidogenesis and therefore secrete a relatively increased proportion of precursor hormones at various stages of steroidogenesis.

The recent study by Taylor et al. (18) described the development of an LC-MS/MS method for quantitation of 13 steroid hormones in serum and applied this method to different adrenal tumors. With the exception of 17-OH-pregnenolone, all analytes accessible in the method by Taylor et al. were also quantified in the present paper. In comparison to our study, the paper by Taylor et al. investigated a smaller cohort of only 48 adrenal tumors and also included radiologically and biochemically confirmed pheochromocytoma/paraganglioma but only ten ACCs. While the authors did not apply machinelearning techniques to determine optimal marker signatures for differential diagnosis, a relative increase of 11-deoxycortisol, 11-deoxycorticosterone and 17-OHP and androstenedione was noted in ACC compared to ACA, which we found here to be of diagnostic relevance as well.

It must be acknowledged though that our study has some limitations. First, our series of adenoma and carcinoma samples is relatively dominated by malignant tumors unlike in clinical practice. This reflects sample acquisition in a tertiary reference center for adrenal tumors, likely leading to sample bias since not the entire spectrum of adenomas encountered in unselected series is covered.
Second, although our study is the largest one that uses plasma steroidomics for the differential diagnosis of ACC and adenomas, absolute numbers are still relatively small, especially for male patients. Third, we included in our series also ENSAT stage IV tumors, which are frequently associated with clinically more severe steroid phenotypes due to their large tumor mass. This fact, however, also applies to the two series referred to above $(14,18)$ and that by Kerkhofs et al. (15). Fourth, we deliberately excluded aldosterone-producing adenomas from our series. Hence, primary hyperaldosteronism cannot be detected and should be ruled out before applying our model. From our point of view, this pre-defined criterion is justified since most aldosterone-producing adenomas are comparably small in size and often diagnosed during workup of hypertension. In contrast, aldosterone-producing ACC are extremely rare and co-secretion of other steroids may be present. Finally, the sample collection in our study was not standardized. Nevertheless, this can also be judged as major advantage of our series (as random sampling generally better reflects clinical routine).

Overall, we think that the combination of steroid hormone profiling together with computational model development holds great diagnostic value, with similar results both in urine and plasma/serum samples. By using additional information about sex, we found two steroid signatures that only share the two hormones DHEA and androstenedione. It is obvious that these signatures may be limited by the relatively small number of samples in the subgroup of males with ACC that was investigated in this study. The rationale behind our approach was to diagnostically use aberrant extragonadal sex hormone production by ACC, which obviously is confounded by gonadal sex steroids. To fully exploit the potential of LC-MS/MS-based differential diagnostics, we enable the community to collect experience and apply our model for research purposes by providing the script of the logistic regression modeling as supplementary material made available online at https://doi.org/10.17605/OSF. IO/8YGNK.

Classically it is proposed to validate diagnostic procedures in an external cohort or even prospectively. However, the overall low incidence of ACC poses particular problems in developing diagnostic algorithms. In fact, retrospective cohorts may not fully represent the current spectrum of disease because of shifts in clinical presentation in the past, e.g. increasingly frequent diagnosis of ACC upon imaging performed for reasons other than adrenal disease. Furthermore, it has recently been recognized that steroid hormone secretion may be a 
continuum between diseases and steroid hormone profiles may overlap between previously assumed clear cut disease entities (33).

In addition, it is expected that machine-learning techniques will be further developed, and new strategies will be possible. We therefore propose to apply machinelearning techniques continuously to routine samples that are analyzed by steroid hormone profiling. This requires application of strict external quality assessment of steroid quantitation and stringent pre-definition end points, e.g. reference histological examination of surgical specimens or stringent imaging follow-up to exclude malignancy.

In conclusion, our analysis strategy that combines LC-MS/MS with machine-learning techniques identifies a reliable diagnostic signature for diagnosis of ACC. Taking the relevant number of patients under study into account $(n=108)$, our plasma-based steroid signature appears to be robust and will hopefully be useful in clinical care for patients with adrenal tumors, since it is applicable with relative ease due to the more widespread availability of LC-MS/MS techniques and plasma/serum samples. Validation in a larger patient cohort is required but may be accomplished ideally by iterative learning using prospectively collected samples in well annotated patients.

\section{Supplementary data}

This is available online at https://doi.org/10.17605/OSF.IO/8YGNK

\section{Declaration of interest}

Martin Fassnacht is a senior editor of European Journal of Endocrinology. Martin Fassnacht was not involved in the peer review or editorial process for this paper on which he is listed as an author. The other authors declare that there is no conflict of interest that could be perceived as prejudicing the impartiality of this study.

\section{Funding}

This work was supported in part by the Deutsche Forschungsgemeinschaft (DFG) (grant numbers KR4371/1-2, FA466/4-2) within the CRC/TRR 205 'The adrenal, central relay in health and disease', project B16 to Ma $\mathrm{Kr}$ and $M$ F, respectively, and a fellowship of the Comprehensive Cancer Center Mainfranken to Ma Kr. Me Ku and Th Da were supported by the Federal Ministry of Education and Research (BMBF) (grant number 031L0129B). This work has been carried out with the assistance of the Interdisciplinary Bank of Biomaterials and Data of the University Hospital of Würzburg and the Julius Maximilian University of Würzburg (IBDW). The implementation of the IBDW has been supported by the Federal Ministry for Education and Research (Grant number FKZ: 01EY1102).

\section{Acknowledgements}

The authors are grateful to Martina Zink for excellent technical support and Michaela Haaf for coordinating the ENSAT Registry.

\section{References}

1 Fassnacht M, Arlt W, Bancos I, Dralle H, Newell-Price J, Sahdev A, Tabarin A, Terzolo M, Tsagarakis S \& Dekkers OM. Management of adrenal incidentalomas: European Society of Endocrinology Clinical Practice Guideline in collaboration with the European Network for the Study of Adrenal Tumors. European Journal of Endocrinology 2016 175 G1-G34. (https://doi.org/10.1530/EJE-16-0467)

2 Else T, Kim AC, Sabolch A, Raymond VM, Kandathil A, Caoili EM, Jolly S, Miller BS, Giordano TJ \& Hammer GD. Adrenocortical carcinoma. Endocrine Reviews 201435 282-326. (https://doi. org/10.1210/er.2013-1029)

3 Fassnacht M, Kroiss M \& Allolio B. Update in adrenocortical carcinoma. Journal of Clinical Endocrinology and Metabolism 201398 4551-4564. (https://doi.org/10.1210/jc.2013-3020)

4 Boland GW, Lee MJ, Gazelle GS, Halpern EF, McNicholas MM \& Mueller PR. Characterization of adrenal masses using unenhanced CT: an analysis of the CT literature. American Journal of Roentgenology 1998171 201-204. (https://doi.org/10.2214/ajr.171.1.9648789)

5 Szolar DH \& Kammerhuber FH. Adrenal adenomas and nonadenomas: assessment of washout at delayed contrast-enhanced CT. Radiology 1998207 369-375. (https://doi.org/10.1148/ radiology.207.2.9577483)

6 Korobkin M, Giordano TJ, Brodeur FJ, Francis IR, Siegelman ES, Quint LE, Dunnick NR, Heiken JP \& Wang HH. Adrenal adenomas: relationship between histologic lipid and CT and MR findings. Radiology 1996200 743-747. (https://doi.org/10.1148/ radiology.200.3.8756925)

7 Kebebew E, Reiff E, Duh QY, Clark OH \& McMillan A. Extent of disease at presentation and outcome for adrenocortical carcinoma: have we made progress? World Journal of Surgery 200630 872-878. (https://doi.org/10.1007/s00268-005-0329-x)

8 Kerkhofs TM, Verhoeven RH, Van der Zwan JM, Dieleman J, Kerstens MN, Links TP, Van de Poll-Franse LV \& Haak HR. Adrenocortical carcinoma: a population-based study on incidence and survival in the Netherlands since 1993. European Journal of Cancer 201349 2579-2586. (https://doi.org/10.1016/j. ejca.2013.02.034)

9 Terzolo M, Angeli A, Fassnacht M, Daffara F, Tauchmanova L, Conton PA, Rossetto R, Buci L, Sperone P, Grossrubatscher E et al. Adjuvant mitotane treatment for adrenocortical carcinoma. New England Journal of Medicine 2007356 2372-2380. (https://doi. org/10.1056/NEJMoa063360)

10 Berruti A, Grisanti S, Pulzer A, Claps M, Daffara F, Loli P, Mannelli M, Boscaro M, Arvat E, Tiberio G et al. Long-term outcomes of adjuvant mitotane therapy in patients with radically resected adrenocortical carcinoma. Journal of Clinical Endocrinology and Metabolism 2017102 1358-1365. (https://doi.org/10.1210/jc.2016-2894)

11 Megerle F, Herrmann W, Schloetelburg W, Ronchi CL, Pulzer A, Quinkler M, Beuschlein F, Hahner S, Kroiss M, Fassnacht M et al. Mitotane monotherapy in patients with advanced adrenocortical carcinoma. Journal of Clinical Endocrinology and Metabolism 2018103 1686-1695. (https://doi.org/10.1210/jc.2017-02591)

12 Gower DB \& Stern MI. Steroid excretion and biosynthesis, with special reference to androst-16-enes, in a woman with a virilising adrenocortical carcinoma. Acta Endocrinologica 196960 265-275. (https://doi.org/10.1530/acta.0.0600265)

13 Gower DB, Daly JR, Snodgrass GJ \& Stern MI. Steroid excretion and biosynthesis with special reference to C19-delta16-steroids in an infant with a virilizing adrenocortical carcinoma. Acta Endocrinologica 197063 562-576. (https://doi.org/10.1530/acta.0.0630562)

14 Arlt W, Biehl M, Taylor AE, Hahner S, Libe R, Hughes BA, Schneider P, Smith DJ, Stiekema H, Krone $\mathrm{N}$ et al. Urine steroid metabolomics as a biomarker tool for detecting malignancy in adrenal tumors. Journal of Clinical Endocrinology and Metabolism 201196 3775-3784. (https:// doi.org/10.1210/jc.2011-1565) 
15 Kerkhofs TMA, Kerstens MN, Kema IP, Willems TP \& Haak HR. Diagnostic value of urinary steroid profiling in the evaluation of adrenal tumors. Hormones and Cancer 20156 168-175. (https://doi. org/10.1007/s12672-015-0224-3)

16 Vogeser M \& Parhofer KG. Liquid chromatography tandemmass spectrometry (LC-MS/MS) - technique and applications in endocrinology. Experimental and Clinical Endocrinology and Diabetes 2007115 559-570. (https://doi.org/10.1055/s-2007-981458)

17 Monaghan PJ, Keevil BG \& Trainer PJ. The use of mass spectrometry to improve the diagnosis and the management of the HPA axis. Reviews in Endocrine and Metabolic Disorders 201314 143-157. (https://doi.org/10.1007/s11154-013-9240-1)

18 Taylor DR, Ghataore L, Couchman L, Vincent RP, Whitelaw B, Lewis D, Diaz-Cano S, Galata G, Schulte KM, Aylwin S et al. A 13-steroid serum panel based on LC-MS/MS: use in detection of adrenocortical carcinoma. Clinical Chemistry 201763 1836-1846. (https://doi.org/10.1373/clinchem.2017.277624)

19 Di Dalmazi G, Fanelli F, Mezzullo M, Casadio E, Rinaldi E, Garelli S, Giampalma E, Mosconi C, Golfieri R, Vicennati V et al. Steroid profiling by LC-MS/MS in nonsecreting and subclinical cortisolsecreting adrenocortical adenomas. Journal of Clinical Endocrinology and Metabolism 2015100 3529-3538. (https://doi.org/10.1210/ JC.2015-1992)

20 Ripley B, Venables B, Bates DM, Hornik K, Gebhardt A \& Firth D. Support Functions and Datasets for Venables and Ripley's MASS. In http://www.stats.ox.ac.uk/pub/MASS4/, 2018.

21 Bruce P \& Andrew B. Practical Statistics for Data Scientists. O'Reilly Media, Boston, MA, USA 2017.

22 James G, Witten D, Hastie T \& Tibshirani R. An Introduction to Statistical Learning: With Applications in R. Springer, Inc., 2014.

23 Sing T, Sander O, Beerenwinkel N \& Lengauer T. ROCR: visualizing classifier performance in R. Bioinformatics 200521 3940-3941. (https://doi.org/10.1093/bioinformatics/bti623)

24 VanDerWal J, Falconi L, Januchowski S, Shoo L \& Storlie C. Species Distribution Modelling Tools: Tools for Processing Data Associated With Species Distribution Modelling Exercises. https://CRAN.R-project.org/ package=SDMTools, 2014.

25 Shackleton $\mathrm{CH}$. Congenital adrenal hyperplasia caused by defect in steroid 21-hydroxylase. Establishment of definitive urinary steroid excretion pattern during first weeks of life. Clinica Chimica Acta 1976 67 287-298. (https://doi.org/10.1016/0009-8981(76)90337-5)
26 Caulfield MP, Lynn T, Gottschalk ME, Jones KL, Taylor NF, Malunowicz EM, Shackleton CH, Reitz RE \& Fisher DA. The diagnosis of congenital adrenal hyperplasia in the newborn by gas chromatography/mass spectrometry analysis of random urine specimens. Journal of Clinical Endocrinology and Metabolism 200287 3682-3690. (https://doi.org/10.1210/jcem.87.8.8712)

27 Krone N, Reisch N, Idkowiak J, Dhir V, Ivison HE, Hughes BA, Rose IT, O'Neil DM, Vijzelaar R, Smith MJ et al. Genotypephenotype analysis in congenital adrenal hyperplasia due to P450 oxidoreductase deficiency. Journal of Clinical Endocrinology and Metabolism 201297 E257-E267. (https://doi.org/10.1210/jc.20110640)

28 Kamrath C, Hartmann MF, Boettcher C, Zimmer KP \& Wudy SA. Diagnosis of 21-hydroxylase deficiency by urinary metabolite ratios using gas chromatography-mass spectrometry analysis: reference values for neonates and infants. Journal of Steroid Biochemistry and Molecular Biology 2016156 10-16. (https://doi.org/10.1016/j. jsbmb.2015.10.013)

29 Arlt W, Walker EA, Draper N, Ivison HE, Ride JP, Hammer F, Chalder SM, Borucka-Mankiewicz M, Hauffa BP, Malunowicz EM et al. Congenital adrenal hyperplasia caused by mutant $\mathrm{P} 450$ oxidoreductase and human androgen synthesis: analytical study. Lancet 2004363 2128-2135. (https://doi.org/10.1016/S01406736(04)16503-3)

30 Minowada S, Kinoshita K, Hara M, Isurugi K, Uchikawa T \& Niijima T. Measurement of urinary steroid profile in patients with adrenal tumor as a screening method for carcinoma. Endocrinologia Japonica 198532 29-37. (https://doi.org/10.1507/ endocrj1954.32.29)

31 Grondal S, Eriksson B, Hagenas L, Werner S \& Curstedt T. Steroid profile in urine: a useful tool in the diagnosis and follow up of adrenocortical carcinoma. Acta Endocrinologica 1990122 656-663. (https://doi.org/10.1530/acta.0.1220656)

32 Kerkhofs TM, Kerstens MN, Kema IP, Willems TP \& Haak HR. Diagnostic value of urinary steroid profiling in the evaluation of adrenal tumors. Hormones and Cancer 20156 168-175. (https://doi. org/10.1007/s12672-015-0224-3)

33 Arlt W, Lang K, Sitch AJ, Dietz AS, Rhayem Y, Bancos I, Feuchtinger A, Chortis V, Gilligan LC, Ludwig P et al. Steroid metabolome analysis reveals prevalent glucocorticoid excess in primary aldosteronism. JCI Insight 20172 e93136.

Received 27 September 2018

Revised version received 15 November 2018

Accepted 22 November 2018 